\title{
ACCESIBILIDAD UNIVERSAL: UNA EXPERIENCIA DE AULA
}

Psicóloga María del Pilar Pinzón *

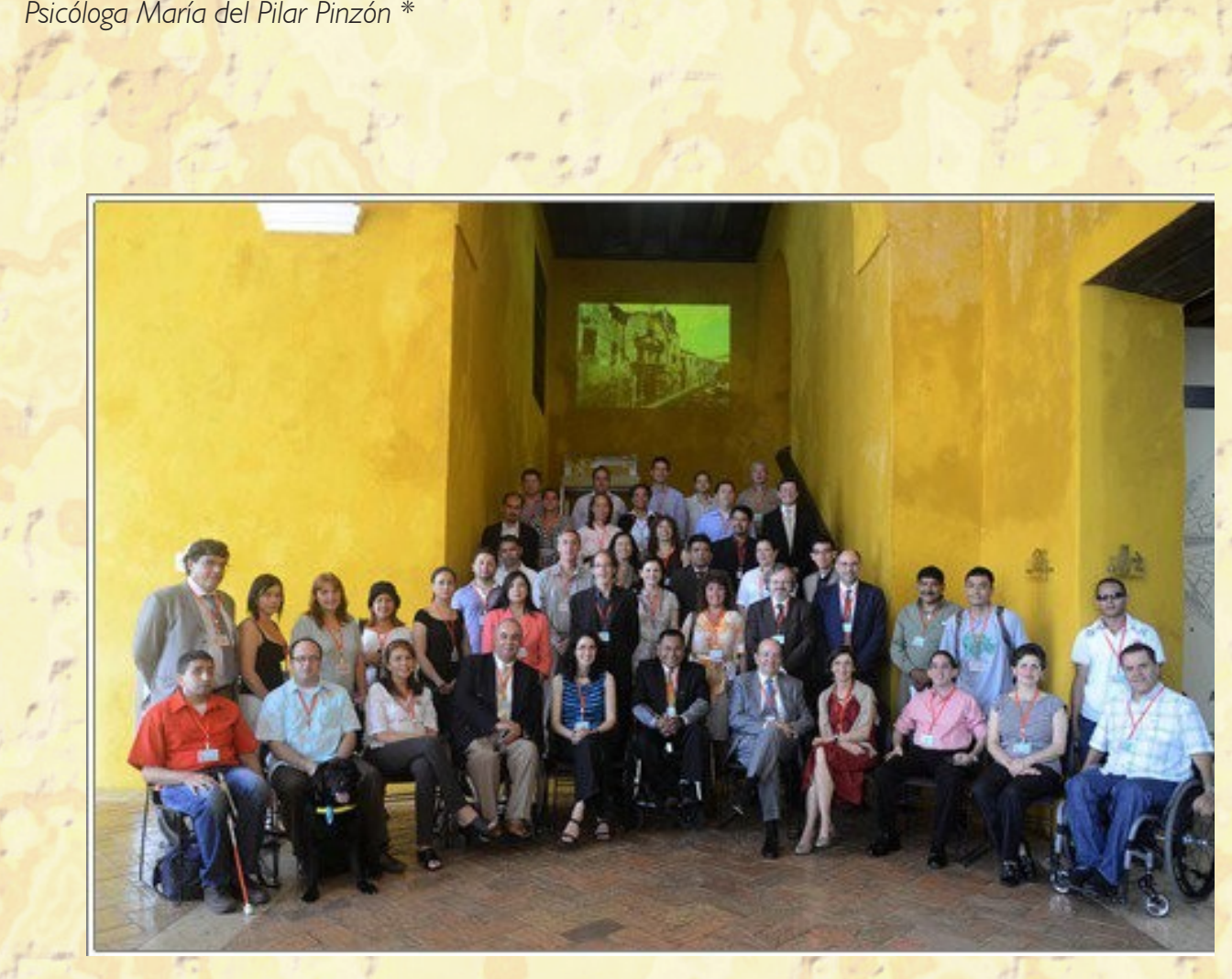

\section{RESUMEN}

La presente ponencia pretende mostrar un breve compendio del recorrido histórico de la Cátedra de accesibilidad implementada en la Facultad de Arquitectura de la Universidad Santo Tomás seccional Bucaramanga, a partir del segundo semestre de 2008; dicha cátedra es una experiencia académica enfocada en el diseño universal tendiente a brindarles a todas las personas independiente de sus condiciones físicas, las garantías para ejercer sus derechos de participación y movilidad en condiciones de igualdad, seguridad y autonomía.

\section{PALABRAS CLAVE}

Accesibilidad, diseño inclusivo, movilidad, discapacidad, inclusión social, diseño universal, entornos incluyentes. 


\section{UNIVERSAL ACCESS: A CLASSROOM EXPERIENCE}

Psicóloga María del Pilar Pinzón

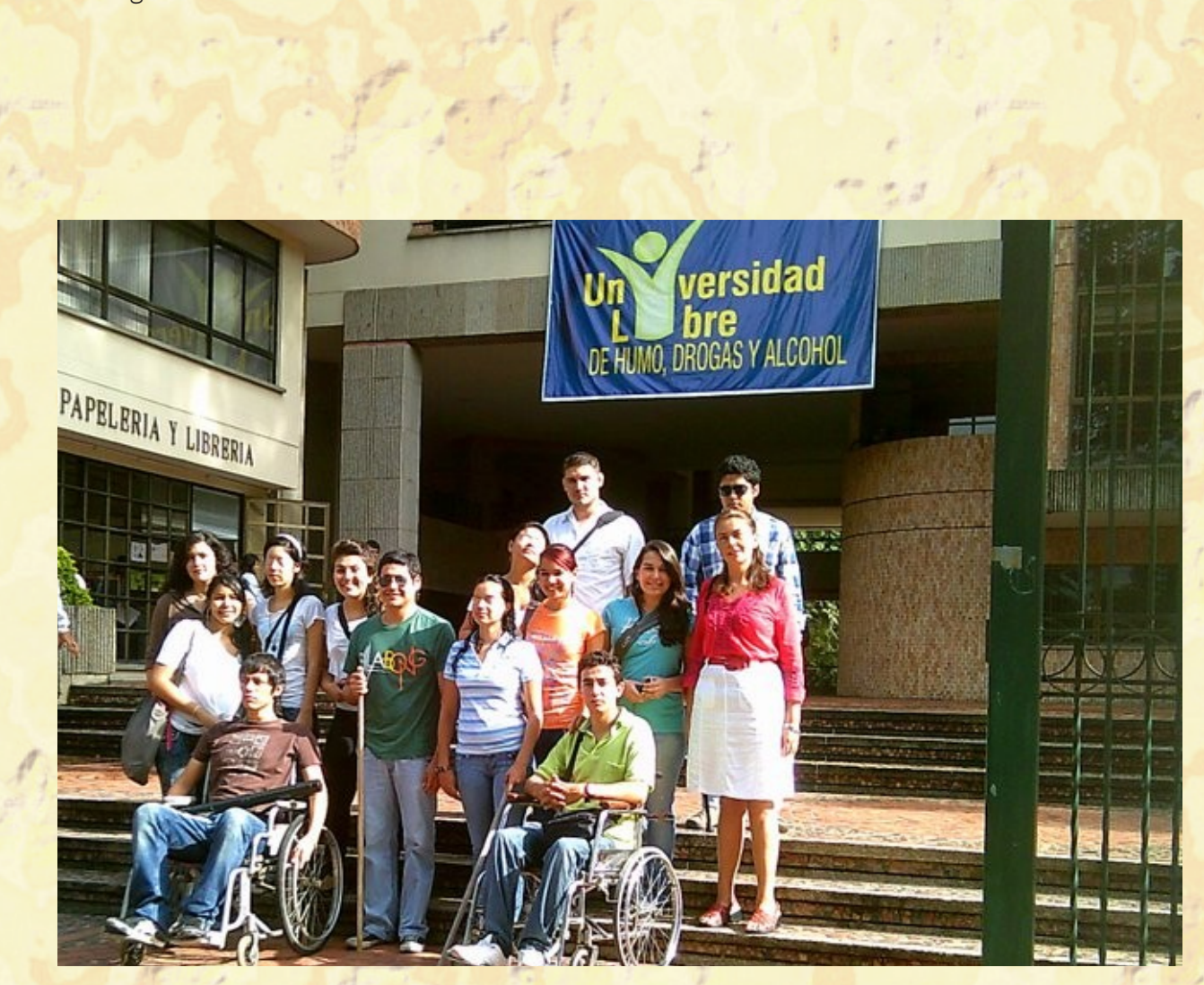

Ponencia presentada en la quinta edición del Seminario Iberoamericano sobre Accesibilidad Universal, organizado conjuntamente por la Agencia Española de Cooperación Internacional para el Desarrollo (AECID), el Real Patronato sobre Discapacidad (Ministerio de Sanidad, Política Social e lgualdad) y la Fundación ACS, en el Centro de Formación de la Cooperación Española en Cartagena de Indias en el mes de Abril de $201 \mathrm{I}$.

* Cátedra Arquitectura Accesible Facultad de Arquitectura, Universidad Santo Tomás, Bucaramanga.

** Con la participación de la Fundación Unicornio. En una primera instancia, la Fundación Unicornio promovió la campaña Bucaramanga Accesible, con el propósito de sensibilizar a la sociedad con respecto a la importancia de la creación de una ciudad más amable para todos en cuanto al diseño y la construcción de espacios incluyentes. A partir de ésta experiencia, se planteó a la Facultad de Arquitectura la posibilidad de implementar allí la cátedra de accesibilidad, para lo cual se contó con el apoyo del Arquitecto Jorge Alberto Villamizar Hernández, quien ocupaba la decanatura al momento de formular la propuesta.

\section{ABSTRACT}

This paper tries to offer a brief overview of the historical overview of the implemented accessibility Chair in the Faculty of Architecture at the University of Santo Tomas Bucaramanga from the second half of 2008 , this chair is an academic experience focused on universal design aimed at provide to all persons regardless of their physical, guarantees to exercise their rights to participation and equal mobility, security and autonomy.

\section{KEY WORDS}

Accessibility, inclusive design, mobility, disability, social inclusion, universal design, inclusive environments. 


\section{PONENCIA}

Desde el segundo semestre de 2008, la cátedra surge por la necesidad ingente de crear espacios formativos donde el arquitecto, no sólo recibiera capacitación en cuanto a normatividad, sino que tuviera participación en un proceso de sensibilización directa, que le permitiera reconstruir un referente social frente al tema de la discapacidad. Uno de los objetivos esenciales es superar los prejuicios y presaberes sociales que apuntan a la discriminación y segregación de la población que tiene limitaciones de movilidad y de acceso a la información.

La facultad de Arquitectura tomó la decisión de incluir la asignatura dentro de su componente flexible en el último trimestre de la carrera, cuya denominación fue Curso Electivo Profesional, asignatura que se mantiene hasta la fecha. De esta forma estudiantes de otras facultades tendrían la opción de tomar la asignatura.

La cátedra parte de la metodología de "Investigación en el aula" a partir del método problémico, desde donde, se plantean dos preguntas iniciales que serán resueltas en el transcurso del semestre:

- ¿Es la ciudad un lugar digno para todos?

- ¿Cómo crear entornos incluyentes que permitan mejorar las condiciones de movilidad y participación de la mayor extensión posible de personas?

Imagen I. Trabajo de Campo SITM Metrolínea. Fuente: Trabajo de campo realizado con los estudiantes de la Facultad de Arquitectura en Febrero de 2011 .

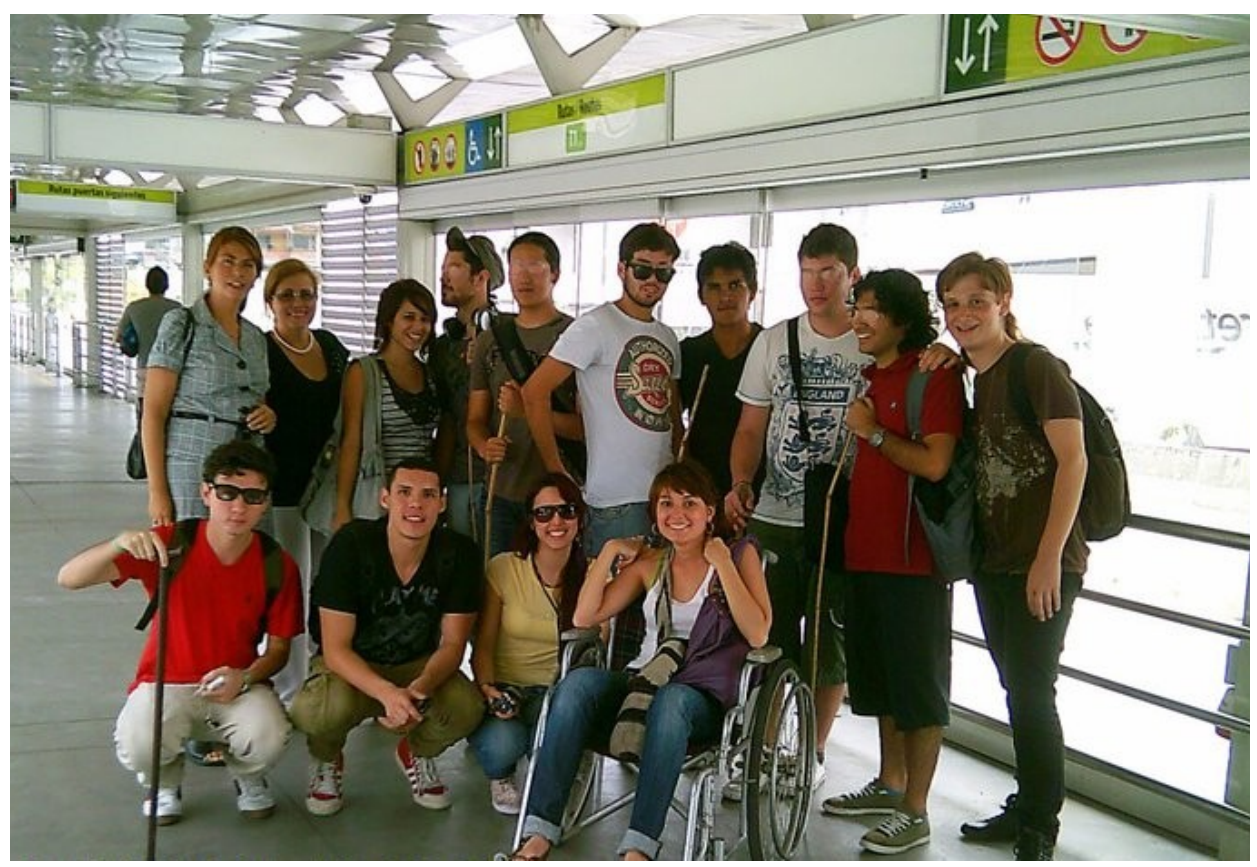




\section{METODOLOGÍA DE LA CÁTEDRA}

La metodología propuesta para la cátedra se desarrolla en cuatro fases, cada una de las cuales se explica a continuación:

Fase de contextualización: el estudiante recibe conceptos teóricos y técnicos relacionados con la normatividad vigente, y aspectos generales de la accesibilidad, el diseño universal, la discapacidad y los problemas de movilidad asociados.

Fase de sensibilización: El estudiante vivencia la situación de discapacidad en recorridos urbanos, evaluando y analizando las condiciones técnicas de construcción urbana y confirmando en terreno la importancia de asumir la normativa como un compromiso de calidad; fortaleciendo su responsabilidad con la construcción de una ciudad más accesible y digna para todos.

Fase de reflexión: El estudiante evalúa y compara lo visto de forma teórica con la realidad práctica de la ciudad.

Fase propositiva: Se plantean mediante acciones propositivas, ciertas preguntas:

- ¿Cómo mejorar las condiciones de movilidad de la mayor cantidad posible de personas en el desarrollo de las actividades vinculadas al espacio público urbano, los medios de transporte y los equipamientos existentes en la ciudad?

- ¿Es posible generar desde el esquema básico, una posición inclusiva y de accesibilidad en los proyectos, sin alterar los elementos estéticos y formales del diseño?

- iSe reeduca con el diseño inclusivo a los usuarios del espacio y su relación cultural con personas en situación de discapacidad?

Como resultados de esta experiencia que lleva desarrollándose desde hace tres años en la Facultad de Arquitectura de manera consecutiva se puede mencionar la presentación de ponencias en el Primer Foro de Arquitectura Accesible USTA 2009, con diferentes propuestas de diseño incluyente. Para el evento fueron invitadas como ponentes, personas en condición de discapacidad. Durante el segundo semestre de 2009 se realizó el Workshop Bucaramanga Maxivalida, experiencia académica interinstitucional de diseño con la participación de estudiantes de arquitectura de la USTA, estudiantes de arquitectura y diseño de la Pontificia Universidad Javeriana y estudiantes de Diseño Industrial de la Universidad Industrial de Santander, donde se mostró un banco de ideas, orientadas a estimular acciones concretas para mejorar las condiciones de movilidad en la ciudad de Bucaramanga. Durante el año 2010 se realizaron dos experiencias de investigación en aula, la primera buscaba reflexionar sobre ¿Es Bucaramanga una ciudad accesible para todos? En la cual los estudiantes visitaron más de 25 lugares abiertos al público de la ciudad de Bucaramanga y su área metropolitana entre los cuales se encontraban, escuelas, universidades, locales comerciales y edificaciones públicas, como alcaldías, notarias, entre otras. De la investigación se pudo concluir que menos del $10 \%$ de las edificaciones de la ciudad de Bucaramanga y su área metropolitana cuentan con condiciones de accesibilidad para todos; a su vez, se realizó una evaluación del Sistema Integral de Transporte Masivo Metrolínea, encontrándose que, si bien su porcentaje de accesibilidad es inferior al $70 \%$ si brinda una mayor movilidad a la población con discapacidad de la ciudad, siendo un referente importante para promover la accesibilidad en el resto de la ciudad. 
En el segundo semestre de 2010 , se realizó una investigación relacionada con el turismo accesible, donde la pregunta de investigación fue: ¿Son los patrimonios turísticos de Santander lugares accesibles para todos? Este trabajo de investigación se realizó en las poblaciones de Girón y Charalá, en donde se analizaron: locales comerciales, establecimientos educativos, parques y edificios públicos e infraestructura turística y hotelera. Una vez procesados los datos levantados tanto en el espacio público como en las edificaciones, se constató, que menos del $10 \%$ de la ciudad de Charalá tiene condiciones de accesibilidad, mientras que Girón tiene un panorama más sobrio, allí tan sólo llega al 5\%, Girón es particularmente complejo dado que es patrimonio histórico y representa el atractivo turístico inmueble más importante del área metropolitana de Bucaramanga.

Algunos de los productos derivados de esta investigación fueron:

- La construcción teórica del proyecto

- Un artículo que resume el trabajo investigación

- Muestras audiovisuales realizadas con el toque juvenil de los estudiantes

\section{CONCLUSIONES}

Las cifras derivadas de las investigaciones, más que alarmarnos, deben servir de motivación para continuar con la tarea de fortalecer procesos como el que actualmente se desarrolla en la Facultad de Arquitectura, donde se ha brindado un apoyo irrestricto a la cátedra. Los ejercicios de Investigación en el Aula, han permitido contar con arquitectos más humanos y sensibles, profesionales e integrales en la construcción de ciudades más justas y equitativas. 
Este primer semestre de 201 I la cátedra continuará afinando estrategias pedagógicas, metodológicas y didácticas, que conduzcan a la formación y sensibilización sistemática sobre la accesibilidad y la construcción de una ciudad más digna para todos. Es un proyecto que sería deseable introducirlo en todas las facultades de arquitectura del país tal y como lo plantea la Ley 36I de febrero de 1997, ley marco de la discapacidad.

\section{BIBLIOGRAFÍA}

Accesibilidad al medio físico y al transporte. Manual de Referencia. Fondo de Prevención vial - Ministerio de Desarrollo Económico - Ministerio de Transporte - Consejería para la Política Social Presidencia de la República. Universidad Nacional de Colombia, Sede Santafé de Bogotá, Facultad de Artes, Oficina de Proyectos. Bogotá, Enero de 2000

CIF, Clasificación Internacional de funcionamiento, OMS, Organización Mundial de la Salud

Compendio de accesibilidad al medio físico. Instituto Colombiano de Normas Técnicas y Certificación ICONTEC, 2000

Decreto 1660 del 16 de Junio de 2003. Ministerio de transporte, República de Colombia

Decreto 1538 del 17 de Mayo de 2005. Ministerio de vivienda y Desarrollo Territorial, República de Colombia

Ley 36 I de 1997. República de Colombia 